Supporting Information for

\title{
Strongly coupled redox-linked conformational switching at the active site of the non-heme iron-dependent dioxygenase, TauD
}

\author{
Christopher W. John, ${ }^{1}$ Greg M. Swain, ${ }^{1}$ Robert P. Hausinger, ${ }^{2,3}$ and Denis A. Proshlyakov ${ }^{1}$ \\ 1 Department of Chemistry, Michigan State University, East Lansing, MI 48824, USA \\ 2 Department of Microbiology and Molecular Genetics, Michigan State University, \\ East Lansing, MI 48824, USA \\ 3 Department of Biochemistry and Molecular Biology, Michigan State University, East \\ Lansing, MI 48824, USA
}

\section{Table of Contents}

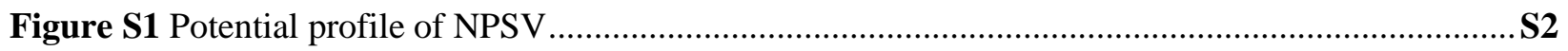

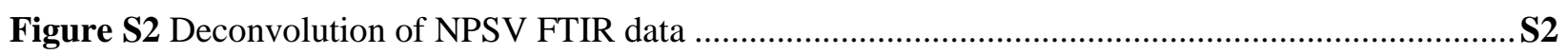

Figure S3 NPSV/GSR resolution of redox transitions in Fe-TauD, 2OG-Fe-TauD, and taurine-2OG-Fe-

TauD

Table S1 Reduction and oxidation potentials of TauD calculated from experimental NPSV profiles ......S3

Figure S4 NPSV/GSR resolution of redox transitions in 2OG-TauD with Zn or Fe bound

Table S2 List of mediators used during NPSV and their thermodynamic and kinetic properties

Table S3 Complete list of mediators tested for NPSV of TauD ...........................................................S5

Figure S5 Graphical representation of the reduced quantitative model. ................................................S5

Figure S6 Titration of 2OG-Fe(II)-TauD into FCN

Figure S7 Extended simulations showing the effect of thermodynamic properties of the analyte on the apparent NPSV redox hysteresis

Table S4 Calculated oxidation and reduction potentials of profiles generated using model [1]

Figure S8 Effect of thermodynamic properties of the analyte on the apparent NPSV redox hysteresis when FCN is not present 


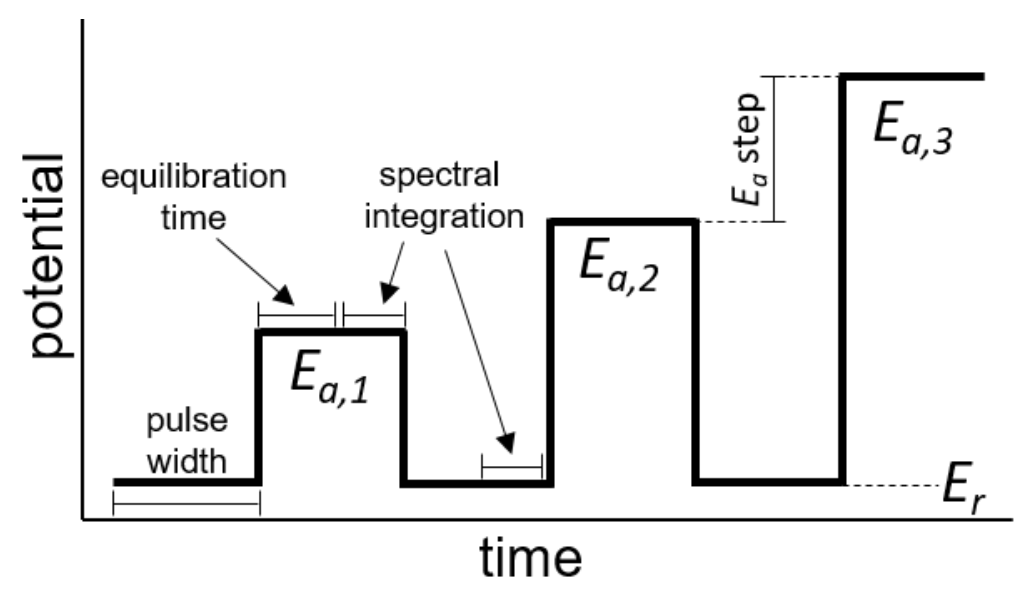

Figure S1. Illustration of the time-dependent profile of potentials used during NPSV. $E_{a}$ alternates between a constant reference, $E_{r}$, and a variable applied potential, $E_{a, i}$, over successive cycles.

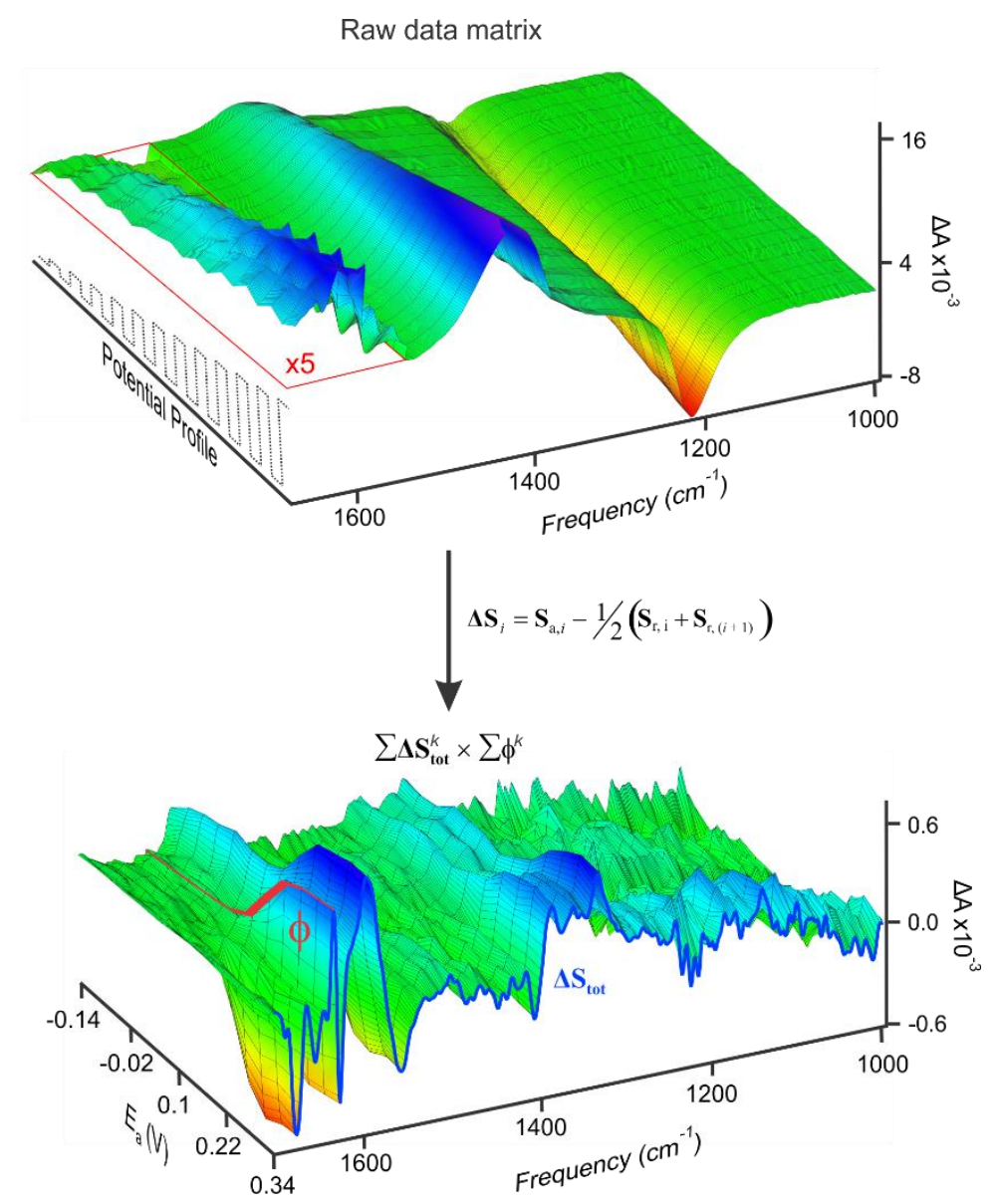

Figure S2. Deconvolution of NPSV FTIR data. Equation (1) was used to process a raw FTIR data (top) into a redox-difference spectral matrix (bottom), which was contributed by all components $(k)$, including redox mediators. These data were further deconvoluted into $\Delta \mathbf{S}_{\text {tot }}$ spectra and $\phi$ profiles of individual components, as shown for TauD by the superimposed blue and red traces, respectively. 


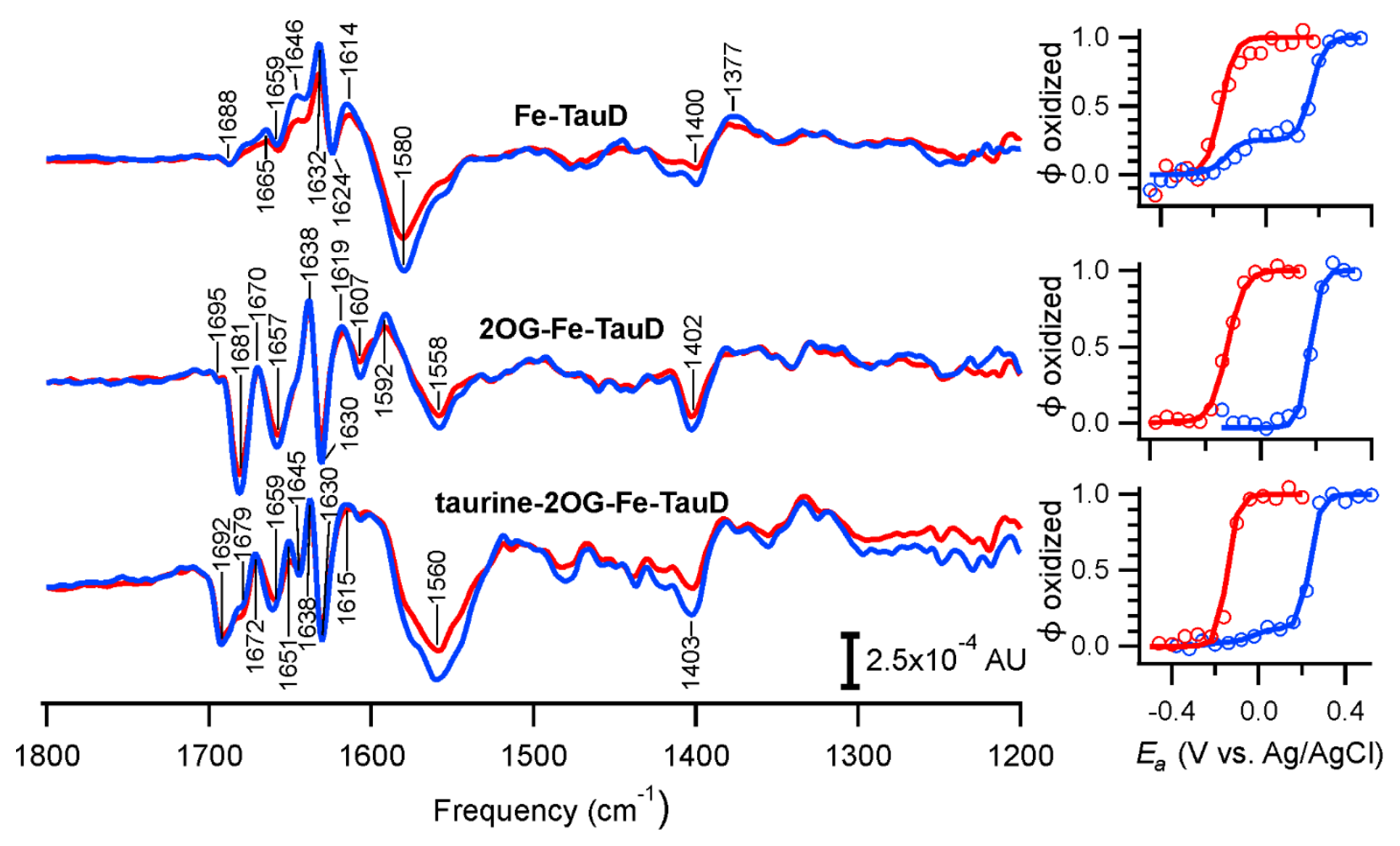

Figure S3. NPSV/GSR resolution of redox transitions in Fe-TauD, 2OG-Fe-TauD, and taurine-2OG-Fe-TauD. Samples were measured in $25 \mathrm{mM}$ Tris, pD 8.5, containing $0.5 \mathrm{M} \mathrm{KCl}$. A mediator cocktail consisting of $100 \mu \mathrm{M} \mathrm{MG}, 100 \mu \mathrm{M}$ TA, and 100 $\mu \mathrm{M}$ FCN was present in all samples. Left: $\Delta S_{t o t}$ of the reduction (-) and oxidation steps (-). Positive modes represent ferric TauD and negative modes represent ferrous TauD. Right: Experimental profiles of reduction steps $(O)$ and oxidation steps $(O)$, normalized using $\Delta A_{1632}^{\text {Fe-TauD }}, \Delta A_{1681}^{\text {2OG-Fe-TauD }}$, and $\Delta A_{1692}^{\text {taurine-2OG-Fe-TauD }}$, and the fitted $\phi$ profiles $(-,-)$.

Table S1. Reduction and oxidation potentials of TauD calculated from experimental NPSV profiles. The maximal magnitude of the observed hysteresis $\left(\Delta E_{1 / 2}\right)$ is shown.

\begin{tabular}{|c|c|c|c|c|}
\hline TauD forms & $\boldsymbol{E}_{\boldsymbol{R} \boldsymbol{d}}(\mathbf{m V})$ & \multicolumn{2}{|c|}{$\boldsymbol{E}_{\boldsymbol{o x}}(\mathbf{m V})$} & \multirow{2}{*}{$\boldsymbol{E}_{1 / 2}(\mathbf{m V})$} \\
& & Phase 1 & Phase 2 & \\
\hline Fe-TauD & $-154 \pm 22$ & $-153 \pm 20$ & $187 \pm 19$ & 341 \\
\hline 2OG-Fe-TauD & $-127 \pm 21$ & - & $171 \pm 17$ & 298 \\
\hline Taurine-2OG-Fe-TauD & $-124 \pm 16$ & $-134 \pm 48$ & $174 \pm 48$ & 298 \\
\hline
\end{tabular}




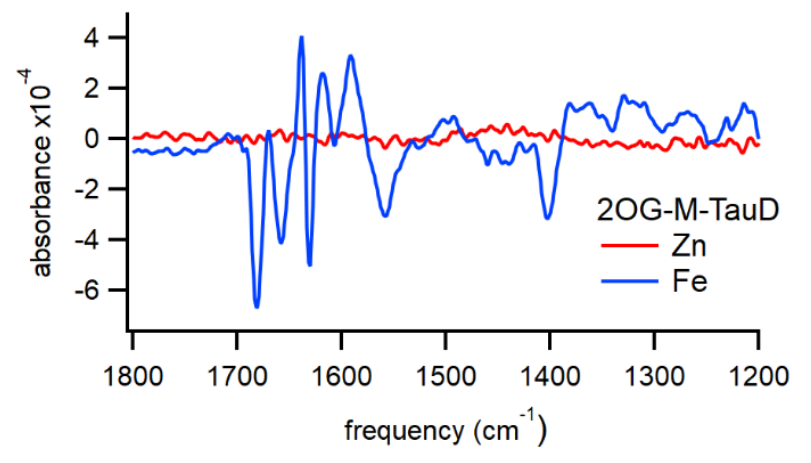

Figure S4. NPSV/GSR resolution of redox transitions when comparing 2OG-TauD with Zn or Fe bound. Both samples were prepared in $25 \mathrm{mM}$ Tris, pD 8.5, containing $0.5 \mathrm{M} \mathrm{KCl}$ and a mediator cocktail consisting of $100 \mu \mathrm{M}$ each of MG, TA, and FCN.

Table S2. List of mediators used during NPSV measurements with their thermodynamic and kinetic properties. $E_{1 / 2}^{1 / 2}$ measured versus a $\mathrm{Ag} / \mathrm{AgCl}$ reference, $n$ is the electron transfer coefficient, $\boldsymbol{k}^{*} \boldsymbol{e l}$ and $\boldsymbol{k}_{\text {lim }}$ are defined separately ${ }^{\mathrm{a}}$, and $\boldsymbol{k}_{\text {sol }}$ is a homogeneous bimolecular rate constant for the reaction with TauD. All values were determined empirically.

\begin{tabular}{|c|c|c|c|c|c|}
\hline Mediator & $\boldsymbol{E}_{1 / 2}(\mathbf{m V})$ & $\boldsymbol{n}$ & $\boldsymbol{k}^{*}{ }^{*}\left(\mathbf{s}^{\mathbf{- 1}}\right)$ & $\boldsymbol{k}_{\text {lim }}\left(\mathbf{s}^{\mathbf{1}}\right)$ & $\boldsymbol{k}_{\text {sol }}\left(\mathbf{M}^{-\mathbf{1}} \mathbf{s}^{-\mathbf{1}}\right)$ \\
\hline FCN & $215^{\mathrm{b}}$ & 1 & 0.1 & 0.2 & $2.0 \times 10^{4}$ \\
\hline MG & $-111,-243$ & 1,1 & 0.025 & 0.5 & $1.4 \times 10^{4}$ \\
\hline TA & $-197^{\mathrm{c}}$ & 2 & 0.5 & 1.88 & $1.7 \times 10^{4}$ \\
\hline
\end{tabular}

${ }^{a}$ John, C. W.; Proshlyakov, D. A. Anal. Chem. 2019, 91, 9563-9570.

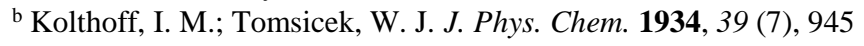

${ }^{c}$ Chen, H. Y.; Zhou, D. M.; Xu, J. J.; Fang, H. Q. J. Electroanal. Chem. 1997, 422 (1-2), 21 
Table S3. Complete list of mediators tested for NPSV of TauD.

\begin{tabular}{|c|c|c|}
\hline Mediator & $\boldsymbol{E}_{1 / 2}(\mathbf{m V})$ & $\boldsymbol{n}$ \\
\hline $\mathrm{FCN}^{\mathrm{a}}$ & 215 & 1 \\
\hline $\mathrm{Ru}\left(\mathrm{NH}_{3}\right)_{6}{ }^{\mathrm{b}}$ & 100 & 1 \\
\hline $2,3,5,6-\mathrm{TMPD}^{\mathrm{c}, \mathrm{d}}$ & 52 & 1 \\
\hline${\mathrm{N}, \mathrm{N}, \mathrm{N}^{\prime}, \mathrm{N}^{\prime}-\mathrm{TMPD}^{\mathrm{c}, \mathrm{d}}}^{\text {indophenol }^{\mathrm{c}}}$ & 25 & 2 \\
\hline $1^{1,2-\text { naphthoquinone }^{\mathrm{c}}}$ & -4 & 2 \\
\hline Fe-EDTA $^{\mathrm{c}}$ & -82 & 1,1 \\
\hline MG $^{\mathrm{T}}$ & $-111,-243$ & 2 \\
\hline methylene blue $^{\mathrm{c}}$ & -197 & 2 \\
\hline methyl viologen $^{\mathrm{c}}$ & -236 & 2 \\
\hline
\end{tabular}

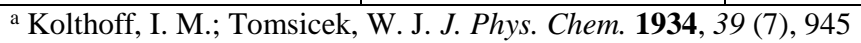

${ }^{\mathrm{b}}$ Meyer, T. J.; Taube, H. Inorg. Chem. 1968, 7 (11), 2369

${ }^{c}$ Fultz, M. Lou; Durst, R. A. Anal. Chim. Acta 1982, 140 (1), 1

${ }^{\mathrm{d}}$ Rawson, F. J.; Downard, A. J.; Baronian, K. H. Sci. Rep. 2015, 4 (1), 5216

${ }^{\mathrm{e}}$ Chen, H. Y.; Zhou, D. M.; Xu, J. J.; Fang, H. Q. J. Electroanal. Chem. 1997, 422 (1-2), 21

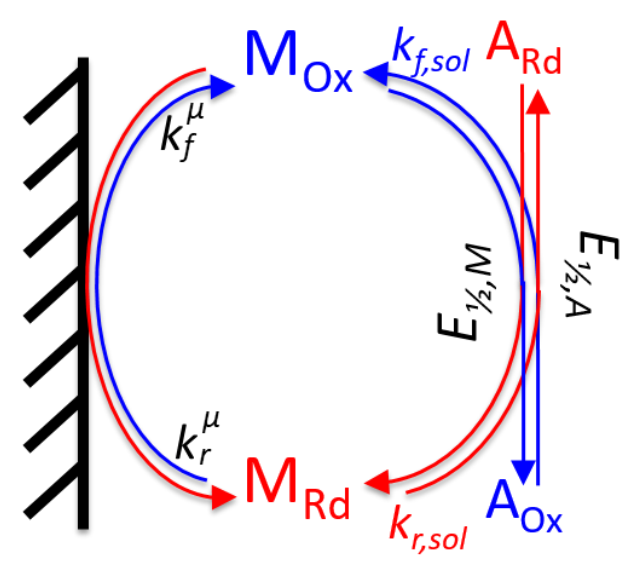

$$
\begin{aligned}
k_{f}^{\mu} & =\frac{1}{\frac{1}{k_{f, e l}}+\frac{1}{k_{l i m}}} \\
k_{r}^{\mu} & =\frac{1}{\left.\left(\frac{1}{k_{r, e l}}+\frac{1}{k_{l i m}}\right) e^{[\alpha n F / R T}\left(E_{a}-E_{1 / 2}\right)\right]}
\end{aligned}
$$

Figure S5. Graphical representation of the reduced quantitative model. M represents the mediator that facilitates electron transfer between the electrode and an analyte, A. The model reduces the electron transfer process of the mediator with the electrode using a reduced rate constant $\left(k_{f}^{\mu}, k_{r}^{\mu}\right)$ that considers both the rate constant of the electron transfer and mass transfer on and off of the electrode $^{1}$ The model also considers the electron transfer between the mediators and analyte $\left(k_{f, s o l}, k_{r, s o l}\right)$, the applied potential at the electrode surface $\left(E_{a p p}\right)$, and $E_{1 / 2}$ of each component.

${ }^{1}$ John, C. W.; Proshlyakov, D. A. FTIR Spectro-Voltammetry and Quantitative Modeling of Analytes in Kinetically Constrained Redox Mixtures. Anal. Chem. 2019, 91, 9563-9570. 


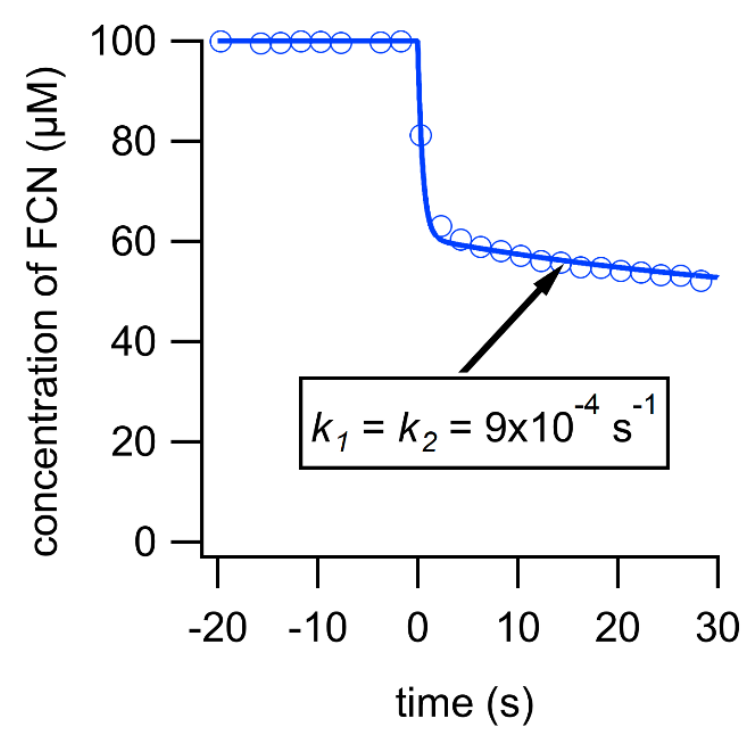

Figure S6. Titration of $70 \mu \mathrm{M}$ 2OG-Fe(II)-TauD into $100 \mu \mathrm{M}$ FCN. The initial electron transfer rate between TauD and FCN was calculated to be $>15,000 \mathrm{M}^{-1} \mathrm{~s}^{-1}$. The value of $k_{2}$, the conversion rate in model [2], is estimated to be $9 \times 10^{-4} \mathrm{~s}^{-1}$ based on the rate of the continued reduction of FCN beginning at about $5 \mathrm{~s}$.

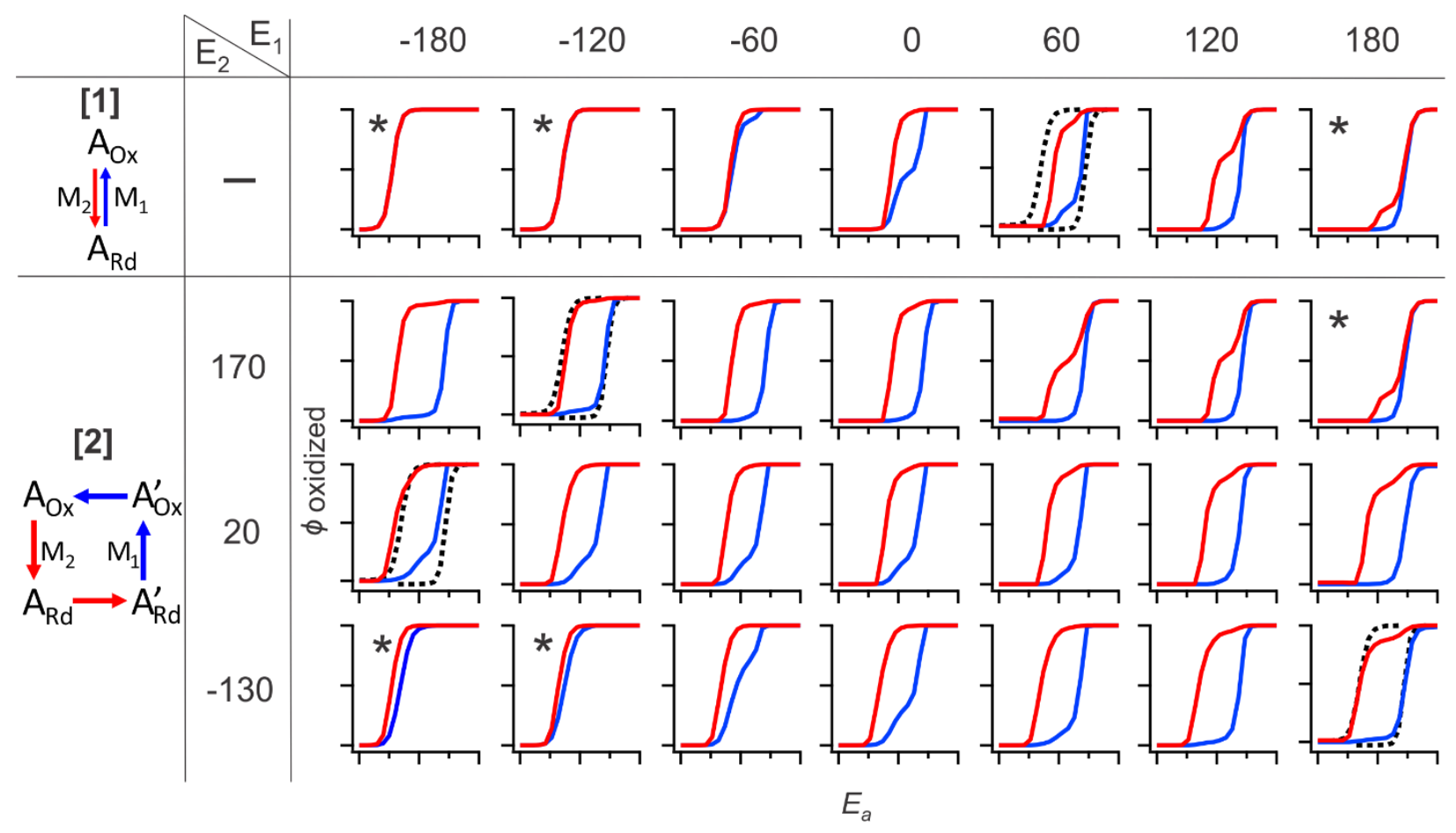

Figure S7. Extended simulations showing the effect of the thermodynamic properties of the analyte on the apparent NPSV redox hysteresis. Population profiles $\phi_{R d}^{A}$ (red) and $\phi_{O x}^{A}$ (blue) were simulated using models [1] (top) and [2] (bottom). Simulations were carried out for a $1 \mathrm{mM}$ analyte using the actual experimental conditions and empirical mediator parameters. The $E_{1 / 2}$ values of the analyte are shown by $E_{1}$ (for model [1]) or $E_{1}$ and $E_{2}$ (for model [2]) in mV. Full scales are $-0.4 \mathrm{~V} \leq E_{a} \leq+0.4 \mathrm{~V}$ and $0 \mathrm{mM}$ $\leq C_{O x} \leq 1 \mathrm{mM}$ for $\mathrm{x}$ and y axes, respectively, for all plots. Population profiles of [2] are the sum of both conformations. Conditions that exhibit predominantly Nernstian behavior are indicated by *. Dashed lines show experimental NPSV profiles of $2 \mathrm{OG}-\mathrm{Fe}-\mathrm{TauD}$ as a reference for selected simulated conditions with comparable levels of hysteresis $\left(\Delta E_{1 / 2}=298 \pm 27 \mathrm{mV}\right)$. 
Table S4. Calculated oxidation and reduction potentials of profiles generated using model [1]. Phases representing $>50 \%$ of the observed oxidation or reduction are bolded. The largest observed hysteresis is italicized.

\begin{tabular}{|c|c|c|c|c|c|c|}
\hline $\begin{array}{c}E_{1 / 2} \\
\text { actual } \\
(\mathbf{m V})\end{array}$ & $\begin{array}{c}E_{O x I} \\
(\mathrm{mV})\end{array}$ & $\begin{array}{c}E_{O x 2} \\
(\mathrm{mV})\end{array}$ & $\begin{array}{l}E_{R d I} \\
(\mathrm{mV})\end{array}$ & $\begin{array}{l}E_{R d 2} \\
(\mathrm{mV})\end{array}$ & $\begin{array}{c}\Delta E_{1 / 2, \max } \\
(\mathrm{mV})\end{array}$ & $\begin{array}{c}\Delta E_{1 / 2, \text { major }} \\
(\mathrm{mV})\end{array}$ \\
\hline 200 & & 200 & 201 & 16.7 & 183 & 1 \\
\hline 180 & & 180 & 187 & -15 & 195 & 7 \\
\hline 160 & & 160 & 174 & -19.5 & 180 & 14 \\
\hline 140 & 126 & 169 & 162 & -27.8 & 197 & 7 \\
\hline 120 & 101 & 164 & 151 & -33.2 & 197 & 197 \\
\hline 100 & 59.5 & 160 & 139 & -37.4 & 197 & 197 \\
\hline 80 & 38.2 & 158 & 127 & -40.5 & 199 & 199 \\
\hline 60 & 18.9 & 156 & 114 & -42.6 & 199 & 199 \\
\hline 40 & 3.55 & 155 & 88.7 & -45.1 & 200 & 200 \\
\hline 20 & -10.1 & 153 & 41.9 & -49.1 & 202 & 202 \\
\hline 0 & -22.6 & 151 & -10.4 & -62 & 213 & 213 \\
\hline-20 & -35 & 146 & -17.3 & -65.6 & 212 & 30.6 \\
\hline-40 & -47.6 & 130 & -17.7 & -70.3 & 200 & 22.7 \\
\hline-60 & -61.3 & 113 & -2.02 & -74.6 & 188 & 13.3 \\
\hline-80 & -78.7 & 113 & & -83.6 & 197 & 4.9 \\
\hline-100 & -99.0 & & & -102 & 3 & 3 \\
\hline-120 & -120 & & & -120 & 0 & 0 \\
\hline-140 & -140 & & & -140 & 0 & 0 \\
\hline-160 & -160 & & & -160 & 0 & 0 \\
\hline-180 & -180 & & & -180 & 0 & 0 \\
\hline-200 & -200 & & & -200 & 0 & 0 \\
\hline
\end{tabular}




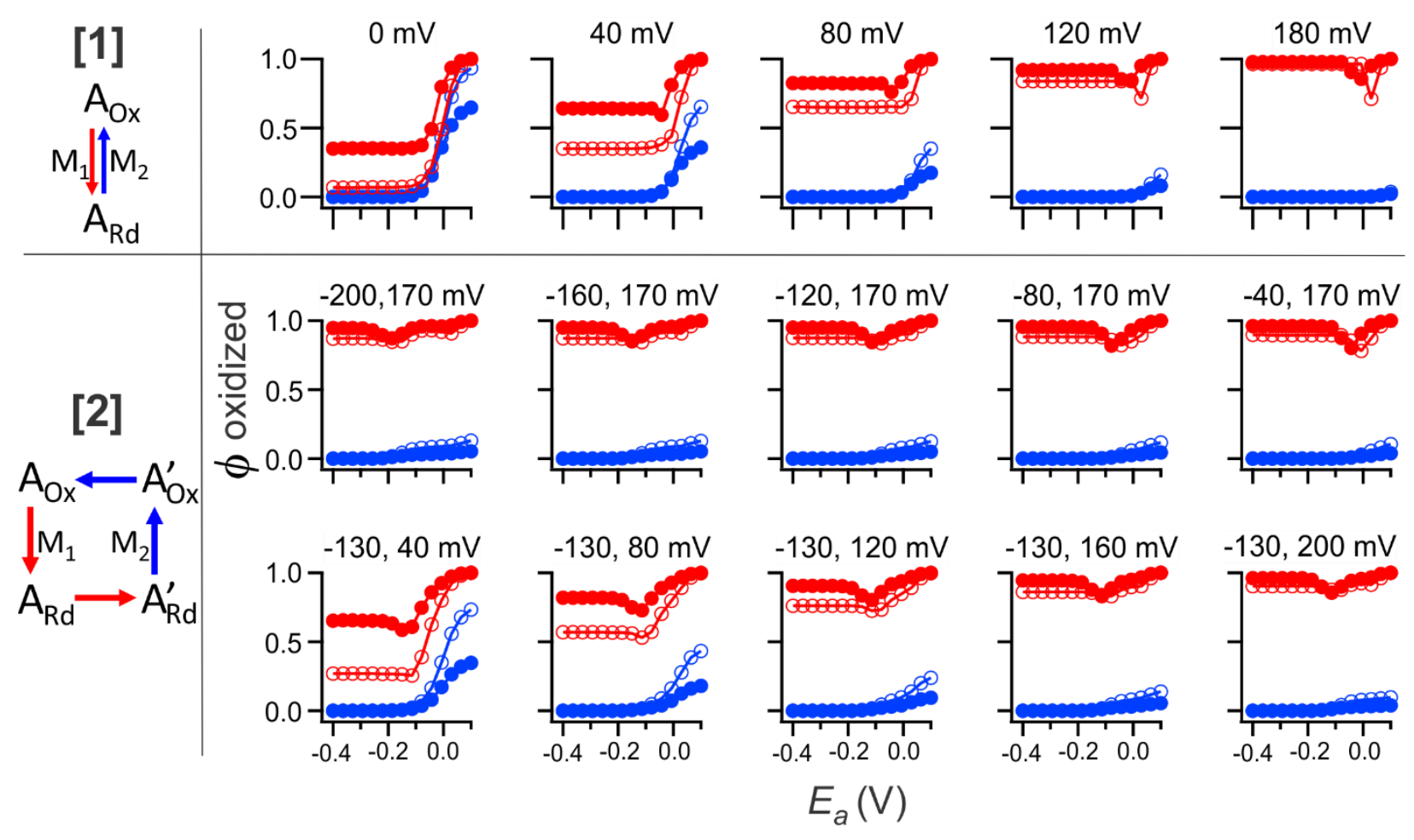

Figure S8. Effect of thermodynamic properties of the analyte on the apparent NPSV redox hysteresis when FCN is not present. Simulations were carried out for $1 \mathrm{mM}$ analyte in the presence of $0.5 \mathrm{mM} \mathrm{MG}$ and TA. The $E_{1 / 2}$ of the analyte is indicated above each simulation set for model [1] and both values are shown for model [2]. Population profiles using model [2] are the sum of both conformations A and A'. Simulations were carried out using a $300 \mathrm{~s}$ pulse width (closed circles) and $1000 \mathrm{~s}$ pulse width (open circles). 